\title{
A COMPARATIVE STUDY OF ANTI-PHOSPHATIDYL INOSITOLE ANTIBODIES IN PATIENTS WITH MYOCARDIAL INFARCTION AND HEALTHY SUBJECTS
}

\author{
${ }^{1,2}$ Abdolreza Sotoodeh Jahromi, ${ }^{1}$ Mohammad Shojaei, \\ ${ }^{3}$ Fatemeh Mehdizadeh, ${ }^{4}$ Saeedeh Erfanian and ${ }^{5}$ Abdolhossien Madani \\ ${ }^{1}$ Research Center for Cardiovascular Atherosclerosis, \\ ${ }^{2}$ Department of Immunology, \\ ${ }^{3}$ Student Research Committee, \\ ${ }^{4}$ Research center for Social determinants of health, \\ Jahrom University of Medical Science, Jahrom, Iran \\ ${ }^{5}$ Research Center for Social Determinants in Health Promotion, \\ Hormozgan University of Medical Science, Bandarabbas, Iran
}

Received 2014-01-14; Revised 2014-01-20; Accepted 2014-01-23

\begin{abstract}
Immune system and inflamation is widely known to play a key role in the development and progression of cardiovascular diseases. Anti-Phospholipid (aPL) antibodies may act in the induction of immunological response leading to the development of Acute Myocardial Infarction (AMI). Anti-Phosphatidyl Inositole (PI) Antibody $(\mathrm{Ab})$ has been seen in various diseases including rheumatoid arthritis, systemic lupus erythematosus and anti-phospholipid antibody syndrome. Although there are a few studies on the association of some autoantibodies with AMI, more epidemiological data are required to confirm their significance as independent risk factors in cardiovascular diseases. Moreover, the data on the relationship of autoantibodies with traditional risk factors of AMI is rare. The study of anti-PI Ab in AMI may lead to understand of etiology of ischemic heart disease. This study was conducted to determine whether prevalence of anti-PI Abs, in patients who had AMI and to analyze their association with traditional cardiovascular risk factors. The prevalence of anti-PI IgG and IgM in a well characterized group of patients with AMI as a case group and in age and sex matched healthy subjects as control group. Sera from the case and the control groups were tested to evaluate the presence of IgG and IgM isotypes to anti-PI by ELISA method. The prevalence of anti-PI IgG and also IgM in the case group resulted significantly higher than in the control group with AMI $(\mathrm{p}<0.005)$. The findings of this study suggest that anti-PI Abs seemed to have a role in AMI independent risk factors for AMI and may represent a link between autoimmunity and atherosclerosis in patients with AMI. Comprehensive studies are recommended to explore the exact role of anti-PI Abs in AMI.
\end{abstract}

Keywords: Anti-Phosphatidyl Inositole (PI) Antibodies, Acute Myocardial Infarction (AMI), AntiPhospholipid (aPL) Antibodies, Cardiovascular Ischemia

\section{INTRODUCTION}

Antiphospholipid Antibodies (Abs) (aPL) in addition to repeated miscarriages and pregnancy complications (Jahromi et al., 2010c; El-Deen, 2013) participate in cardiovascular diseases (Jahromi et al., 2013a; Shojaie and Jahromi, 2011; Jahromi et al., 2013b). However their pathogenic mechanisms are still matter of investigation. Corresponding Author: Mohammad Shojaei, Research Center for Cardiovascular Atherosclerosis, Jahrom University of Medical Science, Jahrom, Iran
In addition to the Classical Lupus Anticoagulant (LAC) and Anti-Cardiolipin Abs (ACA), other antiPhospholipid Abs (aPL) were presented to target anionic phospholipids and other plasma proteins, counting phosphatidyl inositol, protein C, protein S, B2-Glycoprotein I (beta2-gpI) and annexin V (Tincani et al., 2010).

Cardiovascular ischemia is the combined result of environmental factors and personal predispositions. Factors 
such as low serum adiponectin (Shojaie et al., 2009a; Elham et al., 2012) and low annexin V levels (Shojaie et al., 2009b; Youssef et al., 2013) and infectious diseases such as Mycoplasma pneumonia (Pourahmad et al., 2009) are a part of involving factors in AMI. It has been demonstrated that the immunopathological factors such as anti-beta2 glycoprotein-I Ab (Shojaie and Jahromi, 2011) and Inflammatory processes (Jahromi et al., 2010b) have an important role in the initiation and development of AMI.

The results of some studies also demonstrated an association between anti-PL $\mathrm{Ab}$ and Ischemic Heart Disease (IHD) (Shojaie and Jahromi, 2011; Jafarzadeh et al., 2001; Jahromi et al., 2013b; 2013c). It should be noted that genetic factors as well as other traditional risk factors such as smoking, hypercholesterolemia, diabetes mellitus and hypertension may contribute to IHD development and these parameters differ among various population. Although there are a few studies on the association of some autoantibodies with AMI, more epidemiological data are required to confirm their significance as independent risk factors in cardiovascular diseases. Moreover, the data on the relationship of autoantibodies with traditional risk factors of AMI is rare.

Therefore, this case control study was conducted to assess the serum levels of Anti-Phosphatidyl Inositol (PI) Abs (IgG and IgM) in Iranian patients with AMI and healthy subjects, also to elucidate their association with traditional risk factors of the disease.

\section{MATERIALS AND METHODS}

Subjects: A total of 90 consecutive patients (aged 3969 years) with AMI including 67 men and 23 women who were admitted to Peymanieh Hospital of Jahrom in southwest of Iran, were enrolled to this cross-sectional, case-control study.

AMI was diagnosed by the presence of two of the following criteria: (i) prolonged chest pain well-matched with AMI, (ii) typical ECG changes, (iii) rising of cardiac enzymes such as creatine kinase and lactate dehydrogenase (Antman et al., 2004).

Exclusion criteria were valvular heart disease, surgery, trauma during the prior month, cardiomyopathy, liver disease, renal failure, arthritis, malignant diseases, other inflammatory diseases (such as SLE and RA) and oral anticoagulant therapy.

A age, sex and other CAD risk factors such as Hypertension (HTN), Diabetes Mellitus (DM) and Hyper Lipidemia (HLP) matched subjects $(\mathrm{n}=90)$ with similar geographic and socioeconomic backgrounds without any IHD were enrolled as a control group. All control subjects were basically healthy, with no acute or chronic illnesses and did not use any drugs. The healthy control group was recruited from blood donors attending Jahrom Blood Transfusion Center. Peripheral blood (3 cc) was collected from the two groups and the serum was separated and stored at $-20^{\circ} \mathrm{C}$.

The study protocol was permitted by research ethics committee of Jahrom University of Medical Sciences and informed consents were obtained from all participants before enrollment.

Historical, demographic and clinical data were obtained through a review of medical records and interviews with patients and their families. Some risk factors for MI were as follow

I. Age, sex; II. History of hypertension (diagnosis confirmed when the systolic or diastolic pressures were $>160$ or $95 \mathrm{mmHg}$, respectively, or when the patient was using antihypertensive medication); III. Smoking, according to the criteria of the British Council for Medical Research; IV. History of heart disease (atrial fibrillation or coronary heart disease, defined as previous myocardial infarction, angina, or revascularization procedure); V. History of diabetes mellitus, according to the medical history or the use of insulin or an oral anti-diabetes drug; VI. Hypercholesterolemia, based on total cholesterol $>200 \mathrm{mg} \mathrm{dL}^{-1}$, LDL-cholesterol $>130 \mathrm{mg} \mathrm{dL}$, or total cholesterol/HDL-cholesterol ratio >5 (Donahue et al., 1988).

Venous blood samples (3 cc) were collected on admission before starting any IV medications. Isolated sera were frozen within $2 \mathrm{~h}$ after collection and stored at $-20^{\circ} \mathrm{C}$ until laboratory tests.

Serum anti-PI IgG and anti-PI IgM levels was determined by quantitative and qualitative ELISA method respectively, using commercial kit ORGENTEC Diagnostika company, REF: ORG 536, following the manufacturer instructions.

Statistical analyses were performed by SPSS (version 17; SPSS, Inc., Chicago, IL). Data were definited as mean \pm SD. Continuous variables with little-to-mild skewness were summarized as mean \pm SD and compared using Student's t-test.

\section{RESULTS}

Table 1 shows age, sex, laboratory findings and frequencies of each risk factor in the study and the control groups. There was no significant difference between the two groups regarding the following variables: Age, sex, HTN, DM, LDL, HDL, total cholesterol and TG.

In the patient group 16 cases $(18.80 \%)$ had Non-St Elevation MI (NSTEMI) and $74(82.20 \%)$ had ST Elevation MI (STEMI).

The association between positive anti-PI IgG and IgM tests and selected cardiovascular risk factors were assessed. 
Abdolreza Sotoodeh Jahromi et al. / American Journal of Immunology 10 (1): 10-13, 2014

Table 1. Demographic, clinical characteristics and laboratory findings in the case and the control groups

\begin{tabular}{llll}
\hline Variables & Patients group & Control group & P-value \\
\hline Age (year) & $61.64 \pm 9.54$ & $60.49 \pm 10.75$ & 0.612 \\
Male (\%) numbers (\%) & $67(74.40 \%)$ & $(75.60 \%) 68$ & 0.863 \\
High blood pressure (\%) numbers & $39(43.33 \%)$ & $(31.10 \%) 28$ & 0.079 \\
Smoker (\%) numbers & $40(44.40 \%)$ & $(35.60 \%) 32$ & 0.287 \\
IDDM* (\%) numbers & $24(26.70 \%)$ & $(31.10 \%) 28$ & 0.622 \\
Total cholesterol Mg/dL & $176.13 \pm 40.30$ & $168.80 \pm 43.20$ & 0.104 \\
HDL-C* Mg/dL & $44.20 \pm 9.11$ & 0.328 \\
LDL-C* Mg/dL & $118.05 \pm 36.32$ & $98.22 \pm 30.64$ & 0.093 \\
TG $*$ Mg/dL & $126.53 \pm 72.18$ & 0.104 \\
FBS $*$ Mg/dL & $134.20 \pm 54.48$ & $114.84 \pm 41.52$ & 0.495 \\
Positive Anti-PI* IgG (\%) numbers & $25(27.78 \%)$ & $127.08 \pm 53.91$ & 0.006 \\
Positive Anti-PI* IgM $(\%)$ numbers & $9(12.22 \%)$ & $(8.89 \%) 8$ & 0.004 \\
\hline
\end{tabular}

*IDDM = Insuline Dependent Diabetes Mellitus, NIDDM = Non-Insuline Dependent Diabetes Mellitus, HDL-C = High Density Lipoprotein-Cholesterol, LDL-C = Low Density Lipoprotein-Cholesterol, FBS = Fasting Blood Sugar, PI = Phosphatidyl Insositol

Statistically significant associations between positive anti-PI IgG and IgM tests with type hypertension were found, but there was not found any significant association between positive anti-PI IgG and IgM test with type $1 \mathrm{DM}$, type $2 \mathrm{DM}$, age, sex, LDL, HDL, TG, total cholesterol and adjusted smoking.

There was not seen significant difference between positive anti-PI IgG test in patients with STEMI and those with NSTEMI $(73.30$ Vs $25.90 \%),(p=0.186)$ and also there was not found significant difference between positive anti-PI IgM test in patients with STEMI and those with NSTEMI (74.10 Vs $26.70 \%),(p=0.324)$.

\section{DISCUSSION}

The results of the present study indicated that positive anti-PI IgG and also IgM Abs in patients with AMI were significantly more prevalent than that observed in healthy control group. These statistically significant differences were independent of traditional risk factors and consistent with current concepts on the immune pathogenesis of atherosclerosis. However, the precise mechanisms of participation of anti-PI Abs in the pathogenesis of AMI stay to be clarified.

We could not find any research data on anti-PI Abs in AMI for comparing with the results of present study. Other aPLs have been reported to have association with CAD and AMI (Shojaie and Jahromi, 2011; Jafarzadeh et al., 2011; Greco et al., 2010; Urbanus et al., 2009; Jahromi et al., 2013b; 2013c).

There was not found correlation between anti-PI Abs (IgG, IgM) and standard cardiovascular risk factors such as smoking, hypertension and diabetes which is agreement with previous studies (Shojaie and Jahromi, 2011; Jahromi et al., 2010a; 2010b; 2013b; 2013c).
In present study there was a significant association between anti-PI IgG with HTN in the case and the control groups. The same results were found about antiPL IgG in AMI in other studies (Yamada et al., 2010; Jahromi et al., 2013b; 2013c).

As there were not significant differences between positive anti-PI Abs test in patients with STEMI and those with NSTEMI, it can be concluded that anti-PI IgG and IgM do not participate in kind of AMI (STEMI Vs NSTEMI), which is parallel with our previous studies (Jahromi et al., 2013b; 2013c).

\section{CONCLUSION}

Present study may have some limitations. The patients and the healthy subjects were not matched case to case and both groups matched overally.

These results display a statistically significant association between AMI and anti-PI Abs. The results of present study is support by the data from other studies (Shojaie and Jahromi, 2011; Jafarzadeh et al., 2011; Greco et al., 2009; Urbanus et al., 2009; Jahromi et al., 2013b; 2013c) support the potential important role of aPL Abs in AMI.

Comprehensive studies with larger sample size of patients and healthy subjects are recommended to explore the precise role of anti-PI IgG and IgM in IHD.

\section{ACKNOWLEDGEMENT}

This study was financed by Student Research Committee of Jahrom University of Medical Sciences. The authors are grateful to the patients and the control individuals who accepted to enter this study. 


\section{REFERENCES}

Antman, E.M., M. Hand, P.W. Armstrong, E.R. Bates and L.A. Green, 2004. ACC/AHA 2004 Guidelines for the Management of Patients With ST-Elevation Myocardial Infarction: A report of the American College of Cardiology/American Heart Association Task Force on Practice Guidelines: Developed in collaboration With the Canadian Cardiovascular Society endorsed by the American Academy of Family Physicians: 2007 Writing Group to Review New Evidence and Update the ACC/AHA 2004 Guidelines for the Management of Patients With ST-Elevation Myocardial Infarction, Writing on Behalf of the 2004 Writing Committee. Circulation, 15: 296-329.

Donahue, R.P., R.D. Abbott, D.M. Reed, K. Yano, 1988. Physical activity and coronary heart disease in middle-aged and elderly men: The honoluluheart program. Am. J. Publ. Health, 78: 683-685. DOI: 10.2105/AJPH.78.6.683

El-Deen, A., M. Ismail, M.E. El-Gezawy, T. Sherif and K.A. Nasif, 2013. Role of antiphospholipid antibodies in unexplained recurrent abortion and intrauterine fetal death. Life Sci. J., 10: 999-1003.

Elham, O., H. Nayel, A. Zaky and A.K.N. Din, 2012. Serum levels of adiponectin and ghrelin in patients with acute myocardial infarction. Life Sci. J., 9: 523-526.

Greco, T.P., A.M. Conti-Kelly, J.R. Anthony, T. Greco and R. Doyle, 2010. Oxidized-LDL/beta(2)glycoprotein I complexes are associated with disease severity and increased risk for adverse outcomes in patients with acute coronary syndromes. Am. J. Clin. Pathol., 133: 737-743. DOI: 10.1309/AJCP88WVRDRDFBAS

Greco, T.P., A.M. Conti-Kelly, J.R. Greco, R. Doyle and E. Matsuura, 2009. Newer antiphospholipid antibodies predict adverse outcomes in patients with acute coronary syndrome. Am. J. Clin. Pathol., 132: 613-620. DOi: 10.1309/AJCP2FJUT2YZGITK

Jafarzadeh, A., M. Poorgholami, M. Nemati and M. Rezayati, 2011. High serum levels of rheumatoid factor and anti-phosphatidylserine $\mathrm{ab}$ in patients with ischemic heart disease. Iran. J. Immunol., 8: 34-44.

Jahromi, A.S., M. Shojaei, M.R. Farjam and A. Madani, 2013a. Anti-phosphatidylserine antibodies in acute myocardial infarction. Am. J. Immunol., 9: 96-100. DOI: 10.3844/ajisp.2013.96.100

Jahromi, A.S., M. Shojaei, M.R. Farjam and A. Madani, 2013b. Association of anti-phosphatidylcholine antibodies with acute myocardial infarction: A comparative study. Am. J. Immunol., 9: 116-119. DOI: 10.3844/ajisp.2013.116.119
Jahromi, A.S., M. Shojaei, M.R. Farjam and A. Madani, 2013c. The presence of antiphosphatidylethanolamine antibodies in acute myocardial infarction. OnLine J. Biol. Sci., 13: 126130. DOI: 10.3844/ojbsci.2013.126.130

Jahromi, A.S., M. Shojaie and A. Madani, 2010a. Cardiotrophin-1 in patients with acute myocardial infarction. Am. J. Applied Sci., 7: 1190-1194. DOI: 10.3844/ajassp.2010.1190.1194

Jahromi, A.S., M. Shojaie, S. Dana and A. Madani, 2010b. Anti-cardiolipin antibody in acute myocardial infarction. Am. J. Immunol., 6: 11-14. DOI: 10.3844/ajisp.2010.11.14

Jahromi, A.S., M.R. Farjam, F. Mogharrab, A. Daryanavard and A. Madani, 2010c. Anti $\beta 2-$ glycoprotein $\mathrm{i}$ antibodies in women with recurrent spontaneous abortion. Am. J. Biochem. Biotechnol., 6: 264-267. DOI: 10.3844/ajbbsp.2010.264.267

Pourahmad, M., SA. Jahromy, M. Shojaei, 2009. Association of mycoplasma pneumonia infection with myocardial infarction. Am. J. Immunol. 5: 84-88. DOI: 10.3844/ajisp.2009.84.88

Shojaie, M. and A.S. Jahromi, 2011. Anti- $\beta 2$-glycoprotein-i $\mathrm{Ab}$ in acute myocardial infarction. Am. J. Applied Sci., 8: 758-761. DOI: 10.3844/ajassp.2011.758.761

Shojaie, M., A. Sotoodah and G. Shafaie, 2009a. Is adiponectin associated with acute myocardial infarction in Iranian non obese patients. Lipids Health Dis. DOI: 10.1186\%2F1476-511X-8-17

Shojaie, M., A. Sotoodah, S. Roozmeh, E. Kholoosi and S. Dana, 2009b. Annexin V and anti-annexin V antibodies: Two interesting aspects in acute myocardial infarction. Thromb. J., 21: 7-13. DOI: 10.1186/1477-9560-7-13

Tincani, A., C. Casu, S. Cartella, T. Ziglioli and R. Cattaneo, 2010. Antiphospholipid antibody: Laboratory, pathogenesis and clinical manifestations. Reumatismo, 62: 65-75. PMID: 20390120

Urbanus, R.T., B. Siegerink, M. Roest, F.R. Rosendaal and P.G. de Groot, 2009. Antiphospholipid antibodies and risk of myocardial infarction and ischaemic stroke in young women in the RATIO study: A casecontrol study. Lancet Neurol., 8: 998-1005. DOI: 10.1016/S1474-4422(09)70239-X

Yamada, H., T. Atsumi, O. Amengual, T. Koike and I. Furuta, 2010. Anti-beta2 glycoprotein-I antibody increases the risk of pregnancy-induced hypertension: A case-controlled study. J. Reprod. Immunol., 84: 9599. DOI: 10.1016/j.jri.2009.10.005

Youssef, E.M.I., E. El-Shohat, E.S.M. Baiomy, N.F. Abdel and H.N. El-Khouly et al., 2013. Biological value of annexin av and anti-annexin phospholipid (Hughes) syndrome. Life Sci. J., 10: 1144-1148. 